\title{
Titre : Proposition d'un modèle de construction du capital humain en milieu organisationnel
}

Rubrique : Article de recherche

\section{Auteur(s)}

1 : Victor Mignenan

Citation : Mignenan, V. (2020). Proposition d'un modèle de construction du capital humain en milieu organisationnel. Ad Machina, 4(1). doi: 10.1522/radm.no4.1247

\section{Affiliation de l'auteur}

1 : Université : Université du Québec à Chicoutimi_Courriel : victor.mignenan1@uqac.ca

\section{Remerciements}

En premier lieu, je remercie les membres de l'équipe qui ont produit les données qualitatives et quantitatives. En deuxième lieu, mes remerciements s'adressent à toutes les organisations et les personnes qui se sont volontairement prêtées aux entrevues et aux sondages. Enfin, en troisième lieu, je tiens à exprimer mes remerciements à Mlle Christelle Mylène Noutchomwa pour la lecture du texte de cet article.

\section{Déclaration des conflits d'intérêts}

囚 Aucun conflit d'intérêts à déclarer

$\square$ Conflit d'intérêts à déclarer (veuillez détailler)

Détails : 


\title{
Résumé (250 mots)
}

Les études sur le capital humain, menées jusqu’à présent, ont bien révélé son importance pour les organisations. Cependant, sa construction en milieu de travail demeure peu explorée. Même les recherches sur son modèle sont peu nombreuses. Pour comprendre les variables à prioriser, une étude auprès des praticiens et théoriciens tchadiens de GRH a été réalisée. Nous avons appliqué la méthodologie mixte. La production des données a été réalisée au moyen de 11 entrevues semi-structurées et 178 sondages par enquête. L'approche de déconstruction par étape du processus de construction du capital humain, de l'accueil à la permanence, a été utilisée. Les résultats ont montré qu'à l'étape d'accueil du capital humain en milieu organisationnel, les pratiques d'encadrement favorisent les compétences pour exécuter, de façon structurée, les tâches. Ensuite, à l'étape d'intégration, les pratiques d'encadrement et les investissements en formation sont deux leviers d'efficacité. Enfin, à la phase de permanence, nous avons constaté un apport indéniable des investissements en formation, mais ce sont surtout des occasions d'expérience et l'environnement agile qui favorisent la construction de meilleurs talents, en milieu du travail. L'article est utile pour les chercheurs qui trouveront une définition renouvelée du capital humain avec de nouvelles variables et items éprouvés et qui se révèlent plus pertinents. Tandis que les gestionnaires et les consultants trouveront de nouvelles variables permettant de bonifier et valoriser efficacement le capital humain en milieu de travail. Le présent article s'inscrit dans la théorie des ressources et la théorie des compétences, et suggère qu'il existe une contingence relationnelle différenciée à chacune des phases de la construction du capital humain.

\begin{abstract}
The studies on buman capital, carried out to date, have clearly shown its importance for organizations. However, its construction in the workplace remains little explored. Even research on its model is scarce. To understand the variables to be prioritized, a study of Chadian HRM practitioners and theorists was carried out. We applied the mixed methodology. Data was generated through 11 semi-structured interviews and 178 surveys. The step-by-step deconstruction approach of the human capital construction process, from reception to permanence, was used. The results showed that at the stage of welcoming human capital in the organizational environment, coaching practices promote the skills to perform tasks in a structured manner. Then, at the integration stage, coaching practices and investments in training are two effective levers. Finally, during the permanence phase, we have seen an undeniable contribution from investments in training, but it is above all the opportunities for experience and the agile environment that promote the building of the best talents in the workplace. This article is useful for researchers who will find a renewed definition of human capital with new proven variables and items that appear to be more relevant. Managers and consultants will find new variables to effectively enhance and value buman capital in the workplace. This article is in line with the theory of resources and the theory of skills and suggests that there is a differentiated relational contingency in each phase of the construction of human capital.
\end{abstract}

\section{Mots clés}

Formation, agilité, expérience, capital humain, investissement

Droits d'auteur

Ce document est en libre accès, ce qui signifie que le lectorat a accès gratuitement à son contenu. Toutefois, cette œuvre est mise à disposition selon les termes de la licence Creative Commons Attribution (CC BY NC). 


\title{
Proposition d'un modèle de construction du capital humain en milieu organisationnel
}

\author{
Victor Mignenan
}

\section{Introduction}

Toutes les organisations sociales ont du capital humain $(\mathrm{CH})$ (Vinokur, 2014). Bien qu'il varie d'un individu à l'autre, cet ensemble constitué de compétences, savoirs, qualifications ou d'autres capacités qu'un individu possède à des fins productives est indispensable pour la survie d'une organisation. Plusieurs travaux mesurent, habituellement, le $\mathrm{CH}$ par le nombre d'années d'instruction (Becker, 1962; Oxley, Lee et Gibson, 2008; Robinson, Hooker et Mercer, 2008). Tandis que quelques-uns pensent que l'instruction sous-entend une éducation formelle et, par conséquent, cette mesure passe sous silence les autres acquisitions du $\mathrm{CH}$ dont la personne dispose (Bouteiller, Cossette, Fournier et Sabourin, 2013; OCDE, 1998). Les porteurs de cette vision mettent de l'avant les pays sous-développés où l'accès à l'éducation est très hétérogène en matière de qualité, de quantité et de forme. C'est pourquoi quelques auteurs croient que la mesure du $\mathrm{CH}$, exclusivement par le niveau d'instruction, serait biaisée, en partie, à cause de cette hétérogénéité. Cependant, la plupart des auteurs sont d'avis que le $\mathrm{CH}$ demeure un levier important et essentiel pour le développement et la croissance (Antonelli, Antonietti et Guidetti, 2010; Bosma, Praag, Thurik et Wit, 2002). Il constitue un vecteur privilégié pour permettre à l'organisation de réaliser sa mission (Oxley et al., 2008; Robinson et al., 2008). Aussi, permet-il de positionner stratégiquement l'entreprise dans un environnement concurrentiel. C'est pourquoi il est considéré comme la dimension la plus importante du capital intellectuel.

Malgré son importance, le CH n'est pas toujours adéquatement représenté et optimisé en raison de son caractère multidimensionnel. Cette recherche permettra de saisir globalement, dans sa complexité, la problématique de construction du $\mathrm{CH}$, d'en rendre compte, et ainsi d'améliorer sa valorisation. Les théories autour du $\mathrm{CH}$ amènent à penser que ce capital est précieux pour l'entreprise, mais toujours mal corroboré, selon l'expression poppérienne de Mark Blaug (1976). Par conséquent, il demeure une zone grise quant aux modalités de sa construction et à la stratégie pouvant favoriser son optimisation. Ainsi, dans le cadre de cet article, nous prenons acte du succès du $\mathrm{CH}$ au lieu d'insister sur les insuffisances et les présupposés qu'il véhicule. Nous envisageons de contribuer à son renouvellement et sa revalorisation. Dans cette perspective, nous passerons sous silence les perspectives positivistes walrasiennes ou marxistes. Nous adopterons un point de vue aménagé dont les prémisses se trouvent dans l'économie de l'innovation. Pour mener avec succès cette recherche, le fil conducteur se résume à la question suivante : quelles sont les variables jugées pertinentes pour élaborer le meilleur modèle $\mathrm{du} \mathrm{CH}$ au sein d'une organisation? Cette recherche vise à identifier les variables qui entrent dans le processus de construction du $\mathrm{CH}$ en milieu du travail, respectueux du défi actuel de mutation. Sur le plan scientifique, le $\mathrm{CH}$ est un concept à haut potentiel de production scientifique encore mal exploré et optimisé. À travers cette recherche, il sera possible de dessiner un cadre clair et acceptable pour la valorisation endogène du $\mathrm{CH}$.

\section{Mise en contexte théorique}

L'importance du CH a favorisé la construction de sa théorie moderne depuis les années 1960 (Becker, 1962, 2009). La littérature relativement exhaustive a été approfondie pour expliciter ses contenus et mettre en évidence sa portée. Des acteurs individuels et organisationnels ont contribué à la documentation y relative (Becker, 1962; OCDE, 1998; Vinokur, 2014). Pour souligner son importance, l'OCDE (OCDE, 1998) a révélé que l'investissement en capital humain serait la trajectoire pour parvenir à la croissance de l'organisation. D'autres 
travaux enclins à l'essor du $\mathrm{CH}$ postulent que la formation et les occasions d'expérience constituent des leviers pour construire un CH compétent (Bouteiller et al., 2013; Keeley, 2004; Yaroson, Esew et Abdul-Qadir, 2017).

La figure 1 rend compte des trois facteurs régulièrement mis de l'avant pour expliquer le capital humain compétent en milieu organisationnel.

Figure 1

Triangle de construction du capital humain en milieu organisationnel

Source: Données compilées de la littérature (auteur, 2020)

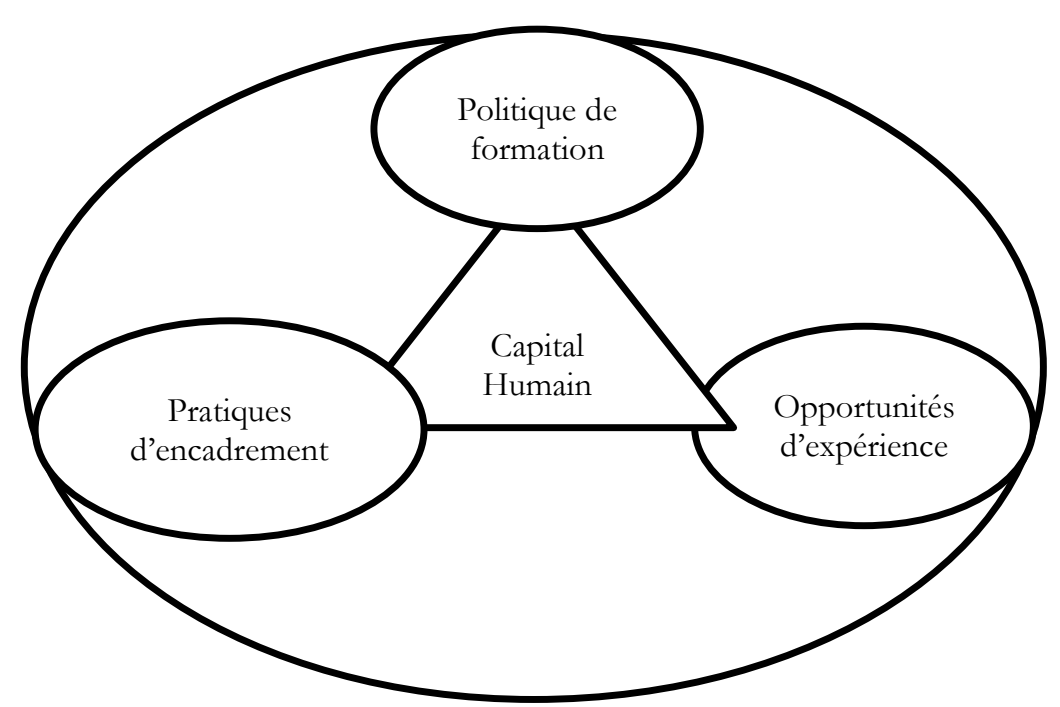

La figure 1 montre que la valorisation du capital humain repose sur trois piliers : la formation, l'encadrement et l'expérience. Il est généralement admis que ce concept est mal fondé, mais se révèle remarquablement fructueux et constitue un meilleur socle de plusieurs recherches théoriques dans les domaines de la répartition (fonctions de gains) et de la croissance (économie du développement et croissance endogène). Cependant, si la notion de $\mathrm{CH}$ est largement comprise, il est plus difficile d'en trouver une définition unanimement reconnue et acceptée par les chercheurs. Dans la séquence qui suit, nous tenterons de suggérer une définition pour notre travail.

\subsection{Capital humain : concept définitionnel}

La formulation du concept « capital humain » n'est pas fortuite. En effet, selon les théoriciens et les praticiens, le capital humain est considéré au même niveau d'importance que le capital financier. Théoriciens et praticiens le considèrent comme une source de richesses et de valeurs (Bouteiller et al., 2013; Keeley, 2004; OCDE, 1998; Yaroson et al., 2017). D’après ces auteurs, le capital humain participe efficacement à l'amélioration de la productivité, mais aussi joue un rôle déterminant dans l'innovation. Tandis que quelques auteurs pensent que le CH ressemble au capital émotionnel (Robinson et al., 2008; Vinokur, 2014) et au capital relationnel. La première dimension est caractérisée par les compétences émotionnelles que possède la personne; il s'agit des capacités favorables au développement personnel, professionnel et organisationnel. La seconde dimension concerne les réseaux relationnels plus ou moins institutionnalisés (Bourdieu, 1980). Selon plusieurs auteurs, le CH d'une organisation peut croître, se réduire, mais aussi devenir inutile si des initiatives pertinentes ne sont pas apportées pour améliorer ses composantes. Malgré son utilité en milieu organisationnel, peu de consensus se dégagent quant au concept définitionnel du capital humain. En effet, selon Joseph Stiglitz (2014), le capital humain est constitué des savoirs, des aptitudes et de l'expérience accumulés qui ont pour effet de rendre les salariés plus 
productifs. Dans le même ordre d'idées, plusieurs auteurs (Ugalde-BindaI, Balbastre-Benavent, Canet-Giner et Escribá-Carda, 2013) déclarent que le $\mathrm{CH}$ est un patrimoine de savoirs à fructifier et non un instrument de production. Il certifie la capacité d'exécution de tâches d'un individu ou d'une population et résulte d'un investissement en éducation et en formation permanente. Rodriguez et Loomis (J. P. Rodriguez et S. R. Loomis, 2007) définissent le capital humain comme un ensemble de savoirs, de connaissances, d'habiletés, de compétences et d'attributs qui participent à la construction du bien-être personnel, économique et social. Selon Becker (1962), le CH est une ressource d'un individu qui détermine son niveau de productivité. Le tableau 1 rend compte de la synthèse définitionnelle.

\section{Tableau 1 \\ Définitions du concept de " capital humain " Source : Données compilées de la littérature (auteur, 2020)}

\begin{tabular}{|c|c|c|}
\hline Auteurs & Définition & $\begin{array}{l}\text { Concept retenu dans le } \\
\text { cadre de ce travail }\end{array}$ \\
\hline $\begin{array}{l}\text { Joseph Stiglitz } \\
\text { (2014), }\end{array}$ & $\begin{array}{l}\text { C’est l'ensemble des compétences et de l'expérience accumulées qui } \\
\text { rendent les salariés productifs. }\end{array}$ & $\begin{array}{l}\text { Compétences et } \\
\text { expérience, salarié plus } \\
\text { productif. }\end{array}$ \\
\hline $\begin{array}{l}\text { P. J. Rodriguez, R.S. } \\
\text { Loomis (2007) }\end{array}$ & $\begin{array}{l}\text { Connaissances, habiletés, compétences et attributs qui facilitent la } \\
\text { création de bien-être personnel, économique et social. }\end{array}$ & $\begin{array}{l}\text { Connaissances, habiletés, } \\
\text { compétences et attributs. }\end{array}$ \\
\hline OCDE (1998) & $\begin{array}{l}\text { Ensemble des connaissances, qualifications, compétences et autres } \\
\text { qualités possédées par un individu et intéressant l'activité économique. }\end{array}$ & $\begin{array}{l}\text { Connaissances, } \\
\text { qualifications, } \\
\text { compétences. }\end{array}$ \\
\hline Gary Becker (1962) & $\begin{array}{l}\text { Ressources d'un individu qui influencent le revenu réel futur généré soit } \\
\text { par l'éducation ou par la formation « on-the-job ». Ici, l'investissement en } \\
\text { capital humain se classifie par son caractère général ou spécifique. }\end{array}$ & Ressources d'un individu. \\
\hline $\begin{array}{l}\text { Theodore Schultz } \\
\text { (1961) }\end{array}$ & $\begin{array}{l}\text { Caractéristiques du travailleur qui déterminent son niveau de } \\
\text { productivité : santé, formation continue pour adulte, éducation, migration } \\
\text { et formation en milieu de travail (ou encore les habiletés motrices, les } \\
\text { méthodes de travail, les connaissances intellectuelles). }\end{array}$ & $\begin{array}{l}\text { Habiletés motrices, } \\
\text { méthodes de travail, } \\
\text { connaissances } \\
\text { intellectuelles. }\end{array}$ \\
\hline
\end{tabular}

Ces définitions reposent, pour l'essentiel, sur les prémisses positivistes walrasiennes et marxistes. Dans le cadre de cet article, le capital humain $(\mathrm{CH})$ est considéré comme une construction conceptuelle et une catégorie de la pratique du rapport de la personne avec le milieu de travail. Dans cette vision, le CH est appréhendé comme «l'ensemble de nouvelles connaissances, compétences, aptitudes, etc. qu'a acquises chaque personne par voie de formation, d'expérience, d'encadrement et d'agilité pendant son cycle de vie professionnelle, lui permettant d'effectuer, de manière structurée, les tâches en milieu de travail. »

\subsection{Cadre théorique du capital humain}

Le capital humain en milieu organisationnel est dilué dans la personne qui l'a construit et l'a acquis. Il est propre à chaque individu dans la mesure où il est le reflet d'une histoire personnelle et d'un parcours singulier relatif au cycle de vie professionnelle. Le processus de développement du capital humain peut provenir de : (i) l'enseignement formel; (ii) la formation en milieu organisationnel; (iii) l'expérience acquise pendant la vie professionnelle dans le vaste ensemble des apprentissages (réseaux d'intérêts, stage d'imprégnation, familles ou communautés); mais aussi de l'environnement agile.

Becker (2009), qui a systématisé la théorie du capital humain, s'est inspiré des travaux fondateurs de Schultz (Schultz, 1961). Le postulat socle de cette théorie repose sur le raisonnement suivant: la richesse de l'Homme porte sur son potentiel de connaissances, de compétences, de savoir-faire, d'intelligence comportementale, etc. Il s'agit d'un véritable capital qui peut être valorisé et enrichi au même titre que le capital matériel. L'organisation qui se dote de la personne comme sujet de croissance doit œuvrer pour faire enrichir non seulement les connaissances de cette dernière, mais aussi son développement dès son entrée 
dans l'entreprise et tout au long de son cheminement avec celle-ci. Selon Becker, il y a deux types d'investissement en capital humain : investissement réalisé en ex ante sur le marché du travail (éducation) et celui fait durant son parcours en milieu du travail (formation continue). Becker constate que la formation initiale de la personne n'est pas suffisante. Par conséquent, l'individu, avec l'apport de son organisation, doit fréquemment faire évoluer (étapes d'accueil à la permanence) ses connaissances de manière à les adapter aux mutations de l'environnement. De plus, les investissements en capital humain peuvent être généraux ou spécifiques. Cependant, tous visent à procurer de nouvelles connaissances, compétences, habiletés liées à des processus techniques ou administratifs qui sont propres à une organisation.

\subsubsection{Apport de la théorie des ressources au capital humain}

La théorie des ressources, qui sous-tend les pratiques managériales, prête des instruments au management stratégique pour la gestion du personnel. Plus spécifiquement, elle incite le replacement du capital humain au centre de la stratégie de l'entreprise. De même, elle favorise la légitimité d'une vision stratégique de la gestion du capital intangible d'une organisation. La théorie des ressources facilite la compréhension de la zone grise entourant la problématique de la performance différenciée durable. Dans la même perspective, Barney révèle que pour déployer des stratégies favorisant l'efficacité et l'efficience, l'organisation est appelée à entretenir ses ressources, notamment son capital humain (Barney, 1991). Pour que cette ressource soit compétitive et durable, l'organisation doit y ajouter de la valeur pour la rendre agile de manière à ce qu'il soit maître d'œuvre de l'innovation stratégique. C'est à cet effet que plusieurs courants de pensée mettent de l'avant l'environnement agile pour expliquer la performance du capital humain. Pour ces auteurs, le $\mathrm{CH}$ étant le plus important de l'ensemble des ressources, il est de la responsabilité de l'organisation de contribuer à sa construction et son développement. Ainsi, la responsabilité du gestionnaire consiste à déployer de meilleures pratiques organisationnelles (management, leadership, gouvernance) pouvant favoriser un environnement agile, mais aussi des investissements conséquents. C'est dans cette perspective qu'émergent des courants de pensée contribuant ainsi à bâtir le capital humain. Il s'agit, entre autres, des perspectives par les connaissances et par les compétences. Ces perspectives ont fait naître la variable éducation-formation dans la théorie du capital humain conceptualisée par Schultz (Schultz, 1961). Selon ce dernier, pour rendre la personne efficace pour le compte de l'organisation, il faut engager des investissements qui génèrent de nouvelles connaissances et expériences.

\subsubsection{Apport de la théorie des compétences au capital humain}

L'idée centrale de la théorie des compétences considère l'organisation comme un ensemble de regroupement des ressources humaines et matérielles dédiées à la production. Il considère le capital humain comme le moyen de production unique et difficilement transférable. Selon la perception de la théorie des compétences (Barney, 1991), le CH incarne des valeurs et patrimoine à fructifier pour être la source d'un avantage compétitif durable (Kungwansupaphan et Siengthai, 2012). C'est dans cette perspective que quelques auteurs (Chamak et Fromage, 2006; Crook, Combs, Todd, Woehr, et Ketchen, 2011; Nafukho, Hairston et Brooks, 2004; Vinokur, 2014) ont d'ailleurs révélé que les connaissances incarnées dans le capital humain sont le patrimoine valorisable et le plus difficile à imiter. Ainsi, ils suggèrent aux entreprises de mettre en place un management "par les compétences », focalisé sur la personne, et recherchant avant tout à accompagner et préparer les employés, patrimoine de croissance, à faire face à un environnement toujours en accélération sous l'effet des technologies de l'information et de la communication.

\subsubsection{Construction du capital humain}

Le capital humain est appréhendé comme un concept singulier. Selon l'avis général, il est mal fondé alors qu'il se révèle extrêmement fructueux pour l'organisation. De même, il offre de nombreuses opportunités de recherches, tant théoriques qu'empiriques. 
Cependant, un consensus interdisciplinaire émerge autour de la construction du capital intellectuel. Ainsi, l'idée d'investir dans les personnes en milieu organisationnel s'est imposée pour répondre aux mutations technologiques et économiques. Cela implique le basculement du modèle d'entreprise capitaliste vers une posture d'entreprise managériale. C'est dans cette perspective qu'Adam Smith, cité par l'OCDE, a constaté que ce qui alimente l'activité économique, ce sont les talents utiles acquis qu'il faut entretenir par des encadrements (Bosma et al., 2002; OCDE, 1998).

Plusieurs auteurs constatent qu'il existe une forte relation de causalité entre les améliorations des pratiques managériales de l'entreprise, les investissements dans les personnes, les encadrements et l'environnement agile, mais aussi l'acquisition d'un capital humain efficace (Kungwansupaphan et Siengthai, 2012; Yaroson et al., 2017). Pour quelques travaux, la politique sociale de l'entreprise devrait donner aux employés peu qualifiés la possibilité de bénéficier des formations continues et de l'environnement agile pour déployer leur talent (Nafukho et al., 2004; J. Rodriguez et S. Loomis, 2007). Toutefois, un certain nombre d'auteurs trouvent que le problème est délicat, car il repose, en partie, sur la structure de l'entreprise (OCDE, 2013; Ugalde-BindaI et al., 2013; Unger, Rauch, Frese et Rosenbusch, 2011). D’autres pensent que le minimum est de créer, au sein de l'entreprise, dès la phase d'intégration, un environnement agile aux nouvelles recrues (Ahmad et Mushraf, 2011; AL-Duajaili, 2012). De même, quelques auteurs trouvent pertinent d'amorcer des formations qualifiantes pour tous les nouveaux salariés afin de leur permettre d'accomplir les tâches de la façon la plus structurée (Lev et Han, 2015).

En guise de synthèse, la figure 2 rend compte de l'articulation entre les principales variables qui jouent un rôle déterminant dans la construction du $\mathrm{CH}$ compétitif.

Figure 2

Composantes du cadre théorique

Source : Données compilées de littérature (auteur, 2020)

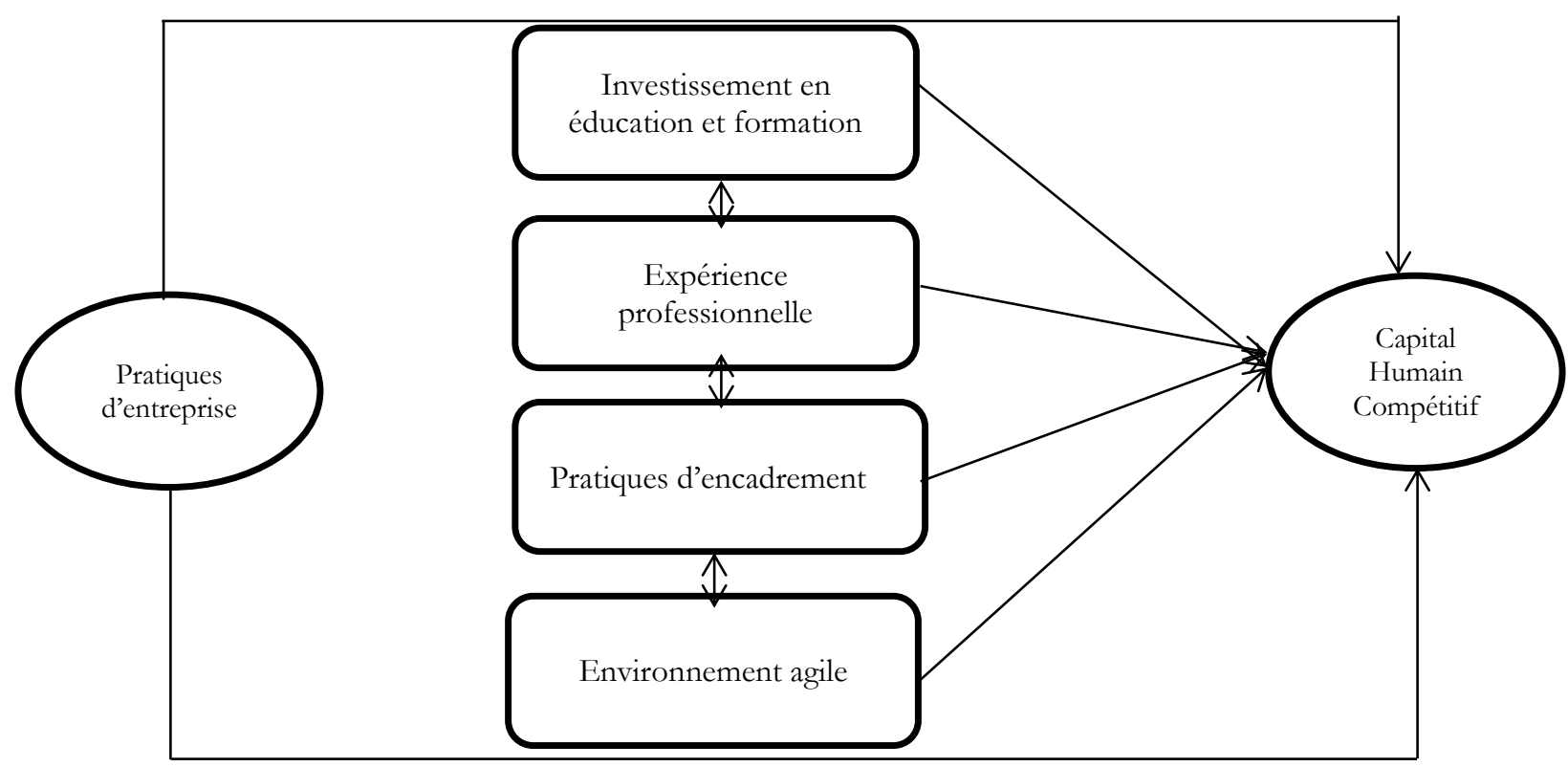

Cette construction du capital humain compétitif (CHC) (figure 2) explicite les interactions entre les pratiques d'entreprise et le $\mathrm{CH}$. Ces relations, qui impriment le modèle, nécessitent des améliorations pour estimer les paramètres. Ces relations portent pour l'essentiel sur les pratiques de l'entreprise en lien avec les différentes facettes du $\mathrm{CH}$, notamment, des pratiques managériales de l'organisation. Ces pratiques 
favoriseront les investissements, l'encadrement, l'expérience et l'agilité comme des facteurs contribuant à la construction du CH. L'articulation de ces variables repose sur le paradigme Structure-Style de management- $\mathrm{CH}$.

\subsection{Cadre conceptuel}

À la lumière de la figure 2, la construction du capital humain de la personne, en milieu de travail, commence le premier jour (étape d'accueil) de la prise de service, par les encadrements/le soutien, et se poursuit jusqu'à la fin du cycle de vie professionnelle (étape de permanence). C'est ainsi que la théorie des ressources et des compétences postule que les investissements en formation, les encadrements, l'expérience et l'agilité, favorisés par le style d'entreprise managériale, contribuent positivement à la construction d'un capital humain compétitif (Becker, 2009; OCDE, 2013; Vinokur, 2014; Yaroson et al., 2017). Cette construction s'enrichit et se développe pendant tout le cycle de la vie professionnelle.

Par conséquent, notre modèle conceptuel de construction du CHC qui est représenté par la figure 3, repose sur le schéma Structure-Pratiques-Résultat.

Figure 3

Modèle conceptuel

Source : Données compilées de la littérature (auteur, 2020)

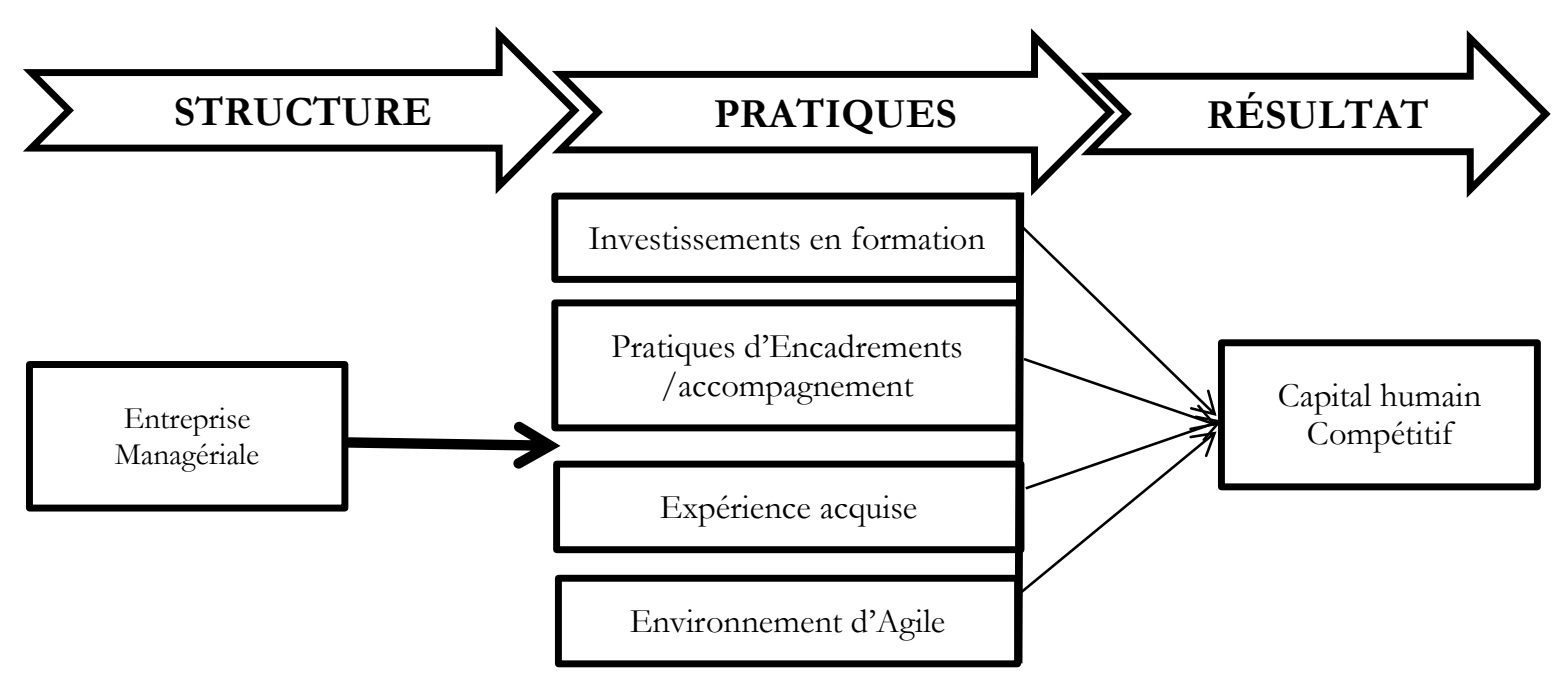

\section{Formulation des hypothèses de recherche}

Notre réflexion est construite autour du postulat selon lequel, grâce à des styles managériaux favorables, les entreprises étudiées déploient des actions pertinentes pour enrichir et développer les nouvelles connaissances et compétences du capital humain qu'elles ont acquises. Nous retenons l'hypothèse générale qui considère le capital humain comme un construit multidimensionnel : il est construit simultanément par les formations, les encadrements, l'expérience, l'environnement agile et, indirectement, par les structures de l'organisation. Quatre hypothèses adjacentes à l'hypothèse générale sont suggérées. Les lignes ci-après rendent compte des quatre hypothèses. 


\subsection{Investissement en formation et capital humain compétitif}

L'investissement en formation consiste à intégrer, dans le budget de l'organisation, une ligne de dépenses dédiées aux activités pouvant enrichir et développer les connaissances du personnel. Les économistes et spécialistes en GRH pensent que l'investissement dans la formation continue des salariés œuvre en faveur de la construction des meilleurs talents (Becker, 1962; Fraisse-D'Olimpio, 2009; Kalkan, Bozkurtb et Arman, 2014; Lleras, 2004; OCDE, 1998; Schultz, 1961; Stiglitz et al., 2014). Les contributions de Becker ont convergé dans la même direction en insistant sur l'importance de la formation par la pratique. Il en ressort ainsi que les investissements dans le capital humain en milieu organisationnel s'inscrivent dans le long terme et que le rôle joué par l'entreprise est déterminant. À la lumière de ce constat, la première hypothèse de recherche ci-dessous est formulée :

Hypothèse 1. Les investissements en formation accroissent la compétence du capital bumain.

\subsection{Pratique d'encadrement et capital humain compétitif (CHC)}

L'encadrement du personnel renvoie aux mécanismes d'accompagnement et de soutien développés au profit des nouvelles recrues. Il consiste à faciliter l'insertion, l'intégration, mais surtout l'exécution des tâches de manière structurée. L'accompagnement est considéré comme l'une des activités efficaces à la construction du $\mathrm{CHC}$ dans le cycle de vie professionnelle. Plusieurs auteurs constatent une influence positive des pratiques d'encadrement et d'accompagnement offertes durant le cycle de vie professionnelle (étapes d'accueil-intégration-permanence) sur l'accroissement de l'habileté du personnel (Crook et al., 2011; Keeley, 2004; Nafukho et al., 2004). Ce qui nous amène à formuler la deuxième hypothèse de la manière suivante :

\section{Hypothèse 2. Les pratiques d'encadrement du personnel améliorent la compétence du capital humain.}

\subsection{Opportunités d'expérience et du capital humain compétitif}

L'expérience est appréhendée en matière de diverses habiletés construites dans l'action et au contact du terrain, du métier, de la fonction ou du poste de travail. Les gestionnaires de RH s'accordent sur le fait que lorsque des opportunités d'accroître et de diversifier les expériences sont créées au profit du personnel, cela favorise l'émergence des talents habiles au sein des travailleurs, dès l'étape d'intégration à la permanence (Becker, 2009; OCDE, 2013; Yaroson et al., 2017). Cette relation nous conduit à suggérer la troisième hypothèse suivante :

Hypothèse 3. Plus l'organisation crée des opportunités d'expérience, plus son capital bumain serait compétent.

\subsection{Environnement agile et capital humain compétitif}

L'agilité est appréhendée comme le mode de gestion du personnel qui accorde de l'importance au travail collaboratif et à l'intelligence collective comme facteur de productivité des individus. La philosophie de l'agilité repose sur l'amélioration continue, l'innovation constante, mais surtout la remise en question des méthodes de manière à répondre véritablement aux attentes des parties prenantes de l'organisation, mais aussi de l'écosystème en perpétuelle mutation. Les approches renouvelées en gestion de projets considèrent l'environnement agile comme facteur associé positivement et statistiquement significatif au CHC. Comme tel, nous suggérons la quatrième hypothèse suivante :

Hypothèse 4. L'environnement agile contribue à la construction du capital bumain compétitif. 
Pour s'assurer que lesdites relations portées par ces variables, sur les plans théorique et managérial, fassent écho aux préoccupations des théoriciens et praticiens, une méthodologie appropriée s'avère indispensable. C’est l'objet de la séquence suivante.

\section{Cheminement méthodologique}

\subsection{Démarche}

Les partisans de la théorie du capital humain s'accordent sur son caractère pluridimensionnel, mais aussi sur son interdisciplinarité. Ainsi, proposer et tester un modèle de construction d'un tel construit en appelle à la mobilisation d'un cadre méthodologique hybride. En conséquence, la démarche mixte (Creswell, 2013), alliant simultanément méthodes qualitatives et quantitatives, a été préconisée.

En effet, dans un premier temps, l'analyse documentaire a été déployée, car elle est la méthode par excellence, non seulement pour appréhender les différentes facettes du capital humain dans son processus de construction, mais pour repérer les variables pertinentes qui y participent. Nous avons ainsi exploré les articles et les rapports. Ces différentes consultations ont permis de : (i) dresser le portrait du capital humain en milieu organisationnel et son processus de construction et; (ii) appréhender les différents courants théoriques autour du capital humain compétitif.

Ensuite, nous avons préconisé l'entretien semi-directif, largement recommandé et utilisé dans le domaine de la science de gestion (Evrard et al., 2009). Dans ce type d'entretien, les répondants disposent d'un éventail de liberté pour fournir l'information détaillée et plus complète. Ainsi, 11 entrevues semi-directives, individuelles, face à face et par téléphone, ont été menées avec chaque chef du personnel ayant volontairement accepté de se prêter aux questions. Ces 11 répondants sont issus de 6 entreprises dans le secteur de la manufacture. Celles-ci ont été sélectionnées en raison de leur engagement dans la mise en place de la politique de responsabilité sociale des entreprises au Tchad. Cet engagement a été favorisé par les recommandations issues du Symposium national sur la responsabilité sociétale des entreprises et le développement durable.

La mise en œuvre de l'entretien est précédée de la construction du guide d'entrevue semi-directive. Ce guide, tenant lieu de véritable fil conducteur, a été construit sur la base des informations générées de la littérature. Il est structuré suivant les quatre thématiques ci-après :
$\checkmark$ Thème 1 : Investissements en formation continue.
$\checkmark$ Thème 2 : Pratiques d'encadrement du personnel.
$\checkmark$ Thème 3 : Opportunités d'expérience du personnel.
$\checkmark$ Thème 4 : Environnent agile.

Il convient de relever aussi que nos répondants se sont gracieusement familiarisés avec les quatre thèmes de l'entrevue. Cet avantage a favorisé l'atteinte du seuil de saturation après avoir réalisé les 11 entrevues, majoritairement par convenance, dans la ville de Moundou (Tchad) au courant des mois de novembre à décembre 2019 avec une durée moyenne de 25 minutes par chef du personnel. Le tableau 2 rend compte des caractéristiques des répondants aux entrevues. 
Tableau 2

Caractéristiques des répondants aux entrevues

Source : Données compilées de la littérature (auteur, 2020)

\begin{tabular}{|c|c|c|c|c|c|c|c|}
\hline \multicolumn{3}{|c|}{ Informations sur les entreprises } & \multicolumn{5}{|c|}{ Informations sur les interviewés } \\
\hline Statut & Secteur & $\begin{array}{l}\text { Taille } \\
\text { moyenne }\end{array}$ & $\begin{array}{l}\text { Date/période } \\
\text { d'entrevue }\end{array}$ & $\begin{array}{l}\text { Durée } \\
\text { moyenne }\end{array}$ & $\begin{array}{r}\text { Ancienneté } \\
\text { dans le poste }\end{array}$ & Catégorie & $\begin{array}{l}\text { Nombre des } \\
\text { interviewés }\end{array}$ \\
\hline $\begin{array}{c}\text { Société à } \\
\text { responsabilité } \\
\text { limitée }\end{array}$ & Produits laitiers & 51 & \multirow{4}{*}{$\begin{array}{c}\text { Novembre à } \\
\text { décembre } \\
2019\end{array}$} & $\begin{array}{c}26 \\
\text { minutes }\end{array}$ & $7-15$ & $\begin{array}{l}\text { Chef du } \\
\text { personnel }\end{array}$ & 6 \\
\hline $\begin{array}{c}\text { Société } \\
\text { anonyme }\end{array}$ & Huile, boissons & 212 & & $\begin{array}{c}28 \\
\text { minutes }\end{array}$ & $5-11$ & $\begin{array}{c}\text { Directeur des } \\
\text { ressources } \\
\text { humaines }\end{array}$ & 3 \\
\hline $\begin{array}{c}\text { Groupement } \\
\text { d'intérêt } \\
\text { économique }\end{array}$ & $\begin{array}{c}\text { Pâtes } \\
\text { alimentaires et } \\
\text { farine }\end{array}$ & \multirow[t]{2}{*}{37} & & $\begin{array}{c}22 \\
\text { minutes }\end{array}$ & $4-9$ & Gestionnaire & 2 \\
\hline & & & & & & & 11 \\
\hline
\end{tabular}

En ce qui concerne la technique d'analyse, nous avons emprunté l'analyse lexicale et de contenu thématique. Le tableau 3 montre comment les deux techniques d'analyse qualitative ont été déployées. Ensuite, une grille d'analyse a été développée suivant les quatre thèmes issus du guide d'entrevue initialement élaboré pour le besoin. Cette grille a fait l'objet d'un remplissage suivant le code des interviewés et des unités d'analyse, lesquels sont soumis à des analyses verticale et horizontale, assorties d'un tableau de synthèse.

Tableau 3

Techniques d'analyse

Source : Inspiré d'Evrard et al., 2005

\begin{tabular}{|l|l|l|}
\hline Nature d'analyse qualitative & Composantes & Indicateurs de pertinence \\
\hline Lexicale & $\begin{array}{l}\text { Mots employés, phrases } \\
\text { ressorties }\end{array}$ & $\begin{array}{l}\text { Type et qualité des vocabulaires employés. } \\
\text { Ex. : fréquence d'apparition des mots, nombre moyen de mots par } \\
\text { phrase, etc. }\end{array}$ \\
\hline Thématique & $\begin{array}{l}\text { Phrases, paragraphes, } \\
\text { thèmes }\end{array}$ & $\begin{array}{l}\text { Découpage thématique } \\
\text { Ex. : fréquence d'apparition des thèmes, fréquence d'association }\end{array}$ \\
\hline
\end{tabular}

L'analyse lexicale et de contenu thématique a permis de faire ressortir de nouvelles facettes de la construction du capital humain compétitif en milieu de travail. Aussi, les résultats ont permis de confirmer les dimensions de la formation continue, et de l'environnement agile dans le processus de construction du capital humain compétitif au travail. De même, l'analyse lexicale et de contenu a participé au repérage des mots et thèmes fréquemment évoqués par les gestionnaires pour éclairer la construction du capital humain compétitif.

En troisième lieu, il s'agit de la phase explicative reposant sur la démarche hypothético-déductive. Nous avons, préalablement, formulé quatre propositions de recherche à partir de la revue de littérature. Le choix du raisonnement hypothético-déductif est motivé par l'immensité de connaissances sur le capital humain, lesquelles sont remarquablement enrichies par les entrevues semi-directives. La production des données s'est réalisée au moyen d'enquêtes menées en face à face ou en ligne (sur une échelle de Likert de cinq points), de décembre 2019 à mars 2020. La formulation des items a précédé l'élaboration du questionnaire, lequel est composé essentiellement des variables qui émergent et des comptes rendus intégraux commentés par quelques spécialistes et professionnels (cabinet d'étude en GRH) complétés par l'exploitation de la 
documentation issue de la littérature. Le questionnaire élaboré a fait l'objet d'un prétest auprès de 14 gestionnaires du personnel et directeurs des bureaux d'études en ressources humaines. Ces répondants ont été choisis sur la base de critères d'expertise en gestion du capital humain.

Pour déterminer la taille de l'échantillon, nous avons emprunté le cheminement recommandé par Igalens et Roussel (1998), qui suggèrent que la taille de l'échantillon est proportionnelle au nombre d'items, soit 5 à 10 fois plus de répondants qu'il y a d'items décrivant les construits à l'étude. Au total, 20 items ont été formulés pour mesurer les 5 construits. Ainsi, nous avons 5 x 20 et 10 x 20, soit entre 100 et 200 . Nous avons opté pour la borne élevée, soit $n=200$ répondants, considérés comme des unités déclarantes. Les 200 répondants sont issus de 10 entreprises (d'au moins 20 salariés chacune - selon l'Agence nationale des investissements et des exportations (ANIE) du secteur manufacturier, sélectionnées au hasard dans la base de données de l'ANIE (Anie-Tchad, 2017). Le questionnaire a été envoyé au chef d'entreprise. Les personnes l'ayant rempli étaient des gestionnaires du personnel ou des responsables d'unités administratives de l'entreprise. Après deux rappels, un total de 178 questionnaires a été reçus; 71,2 \% d'entre eux ont été utilisés dans l'analyse. Le tableau 4 présente les caractéristiques des entreprises terrain de la présente recherche.

\section{Tableau 4 \\ Caractéristiques de l'échantillon \\ Données compilées de la littérature (auteur, 2020)}

\begin{tabular}{|c|c|c|c|c|c|c|}
\hline \multirow[t]{2}{*}{$\mathbf{N}^{\circ}$} & \multirow[t]{2}{*}{ Statut } & \multirow[t]{2}{*}{ Secteur } & \multirow[t]{2}{*}{ Taille (N) } & \multicolumn{3}{|c|}{ Échantillon } \\
\hline & & & & Taille & $\begin{array}{c}\text { Niveau } \\
\text { hiérarchique }\end{array}$ & $\begin{array}{l}\text { Expérience } \\
\text { (en année) }\end{array}$ \\
\hline 1 & \multirow[t]{4}{*}{ SARL } & Produits laitiers & 26 & 10 & \multirow{4}{*}{$\begin{array}{l}\text { Techniciens } \\
\text { et cadres } \\
\text { intermédiaires }\end{array}$} & \multirow{4}{*}{1 à 10} \\
\hline 2 & & Confitures de fruits & 25 & 10 & & \\
\hline 3 & & Pâtes alimentaires & 22 & 10 & & \\
\hline 4 & & Boulangerie & 24 & 10 & & \\
\hline 5 & \multirow[t]{4}{*}{ SA } & Huile végétale & 75 & 45 & \multirow{4}{*}{$\begin{array}{l}\text { Manœuvres, } \\
\text { Techniciens, } \\
\text { cadres } \\
\text { intermédiaires }\end{array}$} & \multirow[t]{4}{*}{1 à 15} \\
\hline 6 & & $\begin{array}{l}\text { Boissons alcoolisée et } \\
\text { gazeuse }\end{array}$ & 62 & 35 & & \\
\hline 7 & & Eau minérale & 53 & 30 & & \\
\hline 8 & & Sucrier & 63 & 30 & & \\
\hline 9 & \multirow[t]{2}{*}{ GIE } & Beurre de karité & 27 & 10 & \multirow{3}{*}{$\begin{array}{c}\text { Techniciens } \\
\text { et cadres } \\
\text { intermédiaires }\end{array}$} & \multirow[t]{3}{*}{1 à 9} \\
\hline 10 & & Beurre d'arachide & 29 & 10 & & \\
\hline Total & & & 406 & 200 & & \\
\hline
\end{tabular}

\subsection{Variables et mesures}

Les mesures adoptées dans notre recherche sont basées sur des études qualitatives et quantitatives antérieures (Becker, 2009; Bouteiller et al., 2013; Crook et al., 2011; Yaroson et al., 2017). Nous les avons adaptées au contexte des entreprises tchadiennes. 


\subsubsection{Variables indépendantes}

Nous avons emprunté les indicateurs issus du compte rendu intégral et ceux couramment utilisés dans l'appréciation et la mesure des variables formation, encadrement, expérience, environnement agile (Becker, 1962; Keeley, 2004; Lleras, 2004; OCDE, 1998; Oxley et al., 2008; Poulain, 2001; Robinson et al., 2008; Schultz, 1961) :

$\checkmark$ Pour la formation, nous avons privilégié : (i) la fréquence des formations; (ii) les thématiques de formation; (iii) les résultats, effets et impact sur le $\mathrm{CH}$.

$\checkmark$ En ce qui concerne l'accompagnement/encadrement, nous avons retenu les trois indicateurs suivants : (i) encadrement technique; (ii) accompagnement administratif; (iii) encadrement pédagogique.

$\checkmark$ Pour l'expérience, nous avons opté pour: (i) la richesse du réseautage; (ii) les voyages/missions de travail; (iii) les visites en entreprise.

$\checkmark$ Pour l'environnement agile, l'accent est mis sur : (i) le niveau d'autonomie du personnel; (ii) la tolérance au risque d'échec; (iii) la collaboration synchronisée; (iv) le niveau d'interaction avec la clientèle.

Les variables explorées ont utilisé des échelles de Likert à cinq points (allant de [1] pas du tout d'accord à [5] tout à fait d'accord). La fiabilité alpha (coefficient de Cronbach) des échelles varie de 0,94 à 0,96 (Becker, 2009; Bessieux-Ollier et Walliser, 2010; Bouteiller et al., 2013; Keeley, 2004; Poulain, 2001; Robinson et al., 2008; J. Rodriguez et S. Loomis, 2007).

\subsubsection{Variable dépendante (capital humain compétitif)}

Les indicateurs retenus, étant donné leur régularité dans la littérature, sont: (i) nouvelles connaissances acquises; (ii) intensité de la motivation; (iii) niveau d'esprit créatif; (iv) participation à des objectifs du personnel; (v) amélioration de l'équilibre social; (vi) niveau d'aptitude/habileté du personnel (Nafukho et al., 2004; OCDE, 1998; Oxley et al., 2008; Robinson et al., 2008; Schultz, 1961; Vinokur, 2014; Yaroson et al., 2017). Les réponses ont été obtenues en utilisant une échelle de Likert (allant de [1] pas du tout d'accord à [5] tout à fait d'accord). La fiabilité alpha de l'échelle est de 0,77.

\section{Résultats et discussion}

\subsection{Résultats}

Sur le plan qualitatif, nous avons mis en évidence les comptes rendus intégraux initialement générés. Le tableau 5 présente le portrait de ces comptes rendus. 


\section{Tableau 5 \\ Comptes rendus intégraux}

\begin{tabular}{|c|c|}
\hline $\begin{array}{l}\text { Variables } \\
\text { testées }\end{array}$ & Comptes rendus intégraux \\
\hline $\begin{array}{l}\text { Investissement en } \\
\text { formation }\end{array}$ & $\begin{array}{l}\text { - } \\
\text { - } \\
\text { - Nous formons tout nouveau personnel recruté; } \\
\text { - } \quad \text { Nous payons, chaque année, le 1,8\% de la masse salariale au titre de la formation continue de notre personnel; } \\
\text { Nous avons élaboré un manuel dans lequel, la politique et le plan de formation continue du personnel sont } \\
\text { définis; } \\
\text { - Nous avons signé une convention avec les bureaux d'études en audit social qui assurent deux missions par an; } \\
\text { - Notre personnel est toujours doté de nouvelles connaissances en sécurité au travail; } \\
\text { - } \quad \text { Nous faisons le renforcement des capacités; } \\
\text { - } \quad \text { Nous actualisons les connaissances de notre personnel. }\end{array}$ \\
\hline $\begin{array}{l}\text { Pratiques } \\
\text { d'encadrement }\end{array}$ & $\begin{array}{l}\text { - Nous avons mis en place un dispositif d'encadrement à l'insertion au profit de toutes les nouvelles recrues; } \\
\text { - } \\
\text { - } \\
\text { - Chous les anciens employés jouent le rôle de soutien et d'encadrement; } \\
\text { - } \quad \text { Dans notre slogan, le chef de service est un coach; } \\
\text { - } \quad \text { Au sein de notre entreprise, ily a le service de soutien pour les nouvelles recrues; } \\
\text { - Ici chez nous, on parle coaching pour les nouvelles recrues. }\end{array}$ \\
\hline $\begin{array}{l}\text { Opportunités } \\
\text { d'expérience }\end{array}$ & $\begin{array}{l}\text { - Selon le niveau de responsabilité et la position du salarié, des missions et voyages professionnels sont régulièrement } \\
\text { organisés selon les besoins de l'entreprise. Des stages d'imprégnation sont également diligentés au profit du } \\
\text { personnel technique; } \\
\text { - Les occasions d'expérience sont beaucoup créées en interne; } \\
\text { - } \quad \text { Le tour des différents services est une occasion d'expérience; } \\
\text { - } \quad \text { Nous avons initié des visites d'imprégnation pour certains personnels; } \\
\text { - } \quad \text { Nous avons organisé, dans notre réseau, des forums d'échanges d'expérience. }\end{array}$ \\
\hline Environnement agile & $\begin{array}{l}\text { - } \\
\text { - } \quad \text { Lous promouvons le travail en équipe fait partie de nó valeurs culturelles, car indispensable pour l'apprentissage collectif, la } \\
\text { créativité, l'innovation et la résolution de problèmes; } \\
\text { - } \quad \text { Notre personnel, notamment les agents de vente, les prospects, la force de vente, travaille en équipe pour créer de } \\
\text { la valeur et des avantages; } \\
\text { - } \quad \text { Parfois, dans les équipes, certaines personnes font peu leur travail; } \\
\text { - } \quad \text { Le personnel nous réclame régulièrement de l'autonomie pour des fins d'épanouissement; } \\
\text { - } \quad \text { Pour nous, le travail coopératif est une sorte d'organisation collective du travail; } \\
\text { - } \quad \text { Je pense que le travail collaboratif est une situation de travail collectif; } \\
\text { - } \quad \text { Agilité c'est aussi forte interactivité, mobilité, flexibilité, efficacité, mais disponibilité; } \\
\text { - } \quad \text { Nous préconisons la flexibilité organisationnelle; } \\
\text { - } \quad \text { Selon notre politique, chaque chef de service doit être tolérant vis-à-vis des risques; } \\
\text { - } \quad \text { Nous promouvons l'autonomie organisationnelle dans tous les services. }\end{array}$ \\
\hline
\end{tabular}

L'analyse des comptes rendus intégraux du tableau 5 a permis de repérer de nouveaux concepts qui tiennent lieu de variables émergentes. Le tableau 6 rend compte du résultat de l'exemple. 
Tableau 6

Exemples de comptes rendus intégraux

\begin{tabular}{|c|c|c|}
\hline $\begin{array}{l}\text { Variables } \\
\text { testées }\end{array}$ & Comptes rendus intégraux & $\begin{array}{c}\text { Variables } \\
\text { émergentes }\end{array}$ \\
\hline $\begin{array}{l}\text { Investissements en } \\
\text { formation }\end{array}$ & $\begin{array}{l}\text { Existence de politique de formation par unité administrative; 1,8\% de la masse salariale allouée à } \\
\text { formation du personnel; en moyenne deux à trois formations du personnel par an; partenaire financier de } \\
\text { formation est FONAP (Fonds national d'appui à la formation professionnelle); existence des institutions } \\
\text { (universités, cabinets/ bureau d'études, etc.) qui offrent des services de formation; mais quelquefois, on est } \\
\text { décu. }\end{array}$ & $\begin{array}{l}\text { Renforcement des } \\
\text { capacités, actualisation } \\
\text { des connaissances. }\end{array}$ \\
\hline $\begin{array}{l}\text { Pratiques } \\
\text { d'encadrement }\end{array}$ & $\begin{array}{l}\text { Le service du personnel, en collaboration avec les différentes unités administratives, met en place des ateliers } \\
\text { d'insertion et d'accompagnement/ encadrement au profit de toutes les nouvelles recrues, pendant les trois à } \\
\text { quatre premières semaines de prise de service selon les fonctions et l'expérience antérieures de la nouvelle } \\
\text { recrue. }\end{array}$ & $\begin{array}{l}\text { Soutien des nouvelles } \\
\text { recrues, accompagner } \\
\text { les nouvelles recrues. }\end{array}$ \\
\hline $\begin{array}{l}\text { Opportunités } \\
\text { d'expérience }\end{array}$ & $\begin{array}{l}\text { Selon le niveau de responsabilité, le poste et la fonction du salarié, des missions et voyages professionnels } \\
\text { sont régulièrement organisés selon les besoins de l'entreprise. Des stages d'imprégnation sont également } \\
\text { diligentés au profit du personnel technique }\end{array}$ & $\begin{array}{l}\text { Visites d'imprégnation; } \\
\text { rencontres d'échanges } \\
\text { d'expérience. }\end{array}$ \\
\hline $\begin{array}{l}\text { Environnement } \\
\text { agile }\end{array}$ & $\begin{array}{l}\text { Le travail en équipe fait partie de nos valeurs culturelles, car indispensable pour l'apprentissage collectif, } \\
\text { la créativité, l'innovation et la résolution de problèmes. Des agents de vente, les prospects, la force de vente } \\
\text { travaillent en équipe pour créer de la valeur et des avantages. De fois, dans les équipes, certaines personnes } \\
\text { font peu leur travail. }\end{array}$ & $\begin{array}{l}\text { Flexibilité } \\
\text { organisationnelle; } \\
\text { tolérance aux risques, et } \\
\text { autonomie } \\
\text { organisationnelle. }\end{array}$ \\
\hline
\end{tabular}

Sur le plan corrélationnel, nous avons mis de l'avant les paramètres moyenne et écart-type (Éc.T.). Le tableau 7 rend compte des résultats. Ainsi, d'une manière générale, les parties prenantes du capital humain des entreprises étudiées perçoivent la formation, l'encadrement, l'expérience et l'agilité comme étant des facteurs de construction d'un capital humain compétitif, dès l'étape d'accueil à l'intégration. En effet, elles rapportent que leur organisation investit dans la formation de ses salariés avec une moyenne de 3,9 sur 5 $(E . T .=1,63)$. Elles déclarent amorcer des pratiques d'encadrement, dès l'étape d'accueil, avec la moyenne de 3,5 sur $5(E ́ c . T .=1,45)$. Ensuite, ils mentionnent que leur organisation crée des opportunités d'expérience, lors de l'étape de permanence, avec une moyenne de 3,6 (Éc.T.= 1,53). Enfin, elles soulignent que leur entreprise crée un environnement agile avec une moyenne de 4,8 (Éc.T.= 1,65). Bref, les répondants considèrent leurs organisations comme davantage enclines à la valorisation et l'enrichissement $\mathrm{du} \mathrm{CH}$. Ces résultats sont, certes, tous positifs, et ce, bien que des entrevues font ressortir quelques points de vue divergents, notamment certains dirigeants d'entreprises qui sont peu favorables à la formation continue. Les résultats révèlent aussi que les répondants partagent les efforts consentis par leurs organisations quant aux investissements en $\mathrm{CH}$. En effet, ils soulignent les initiatives dans le domaine de l'agilité, de la formation, etc. comme étant des variables à promouvoir dans le processus de construction du capital humain compétitif. Enfin, le tableau 7 présente aussi les résultats corrélationnels forts entre les variables. Plus spécifiquement, l'environnement agile présente la plus forte corrélation $(r=0,96 ; p<, 01)$ et statistiquement positif avec le CHC. L'encadrement/soutien est la deuxième variable fortement corrélée $(r=0,91, \mathrm{p}<, 01)$ statistiquement positive avec le CHC. La formation vient en troisième position avec une corrélation positive et statistiquement significative $(\mathrm{r}=0,89, \mathrm{p}<, 01)$. Enfin, l'expérience est associée positivement au CHC $(r=0,83, \mathrm{p}<, 01)$. Ces résultats convergent avec les comptes rendus intégraux provenant des entrevues semi-directives du tableau 7 , lesquels résultats abondent dans le sens des hypothèses de recherche. 


\section{Tableau 7 \\ Moyennes, écarts-types et corrélations entre les variables}

\begin{tabular}{|c|c|c|c|c|c|c|}
\hline Variables & Moyenne (É.T) & $\begin{array}{l}\text { Capital humain } \\
\text { compétitif }\end{array}$ & Formation & Encadrement & $\begin{array}{c}\text { Expérienc } \\
\mathrm{e}\end{array}$ & Agilité \\
\hline $\begin{array}{l}\text { Capital humain } \\
\text { compétitif }\end{array}$ & $4,9(1,87)$ & 1 & & & & \\
\hline Formation & $3,9(1,63)$ & $0,89 * *$ & 1 & & & \\
\hline Encadrement & $3,5(1,45)$ & $0,91 * *$ & $0,74 * *$ & 1 & & \\
\hline Expérience & $3,6(1,53)$ & $0,83 * *$ & $0,67 * *$ & $0,88^{* *}$ & 1 & \\
\hline Agilité & $4,8(1,65)$ & $0,96 * *$ & $0,81^{* *}$ & $0,56 * *$ & $0,78^{* *}$ & 1 \\
\hline
\end{tabular}

En guise de conclusion, si chaque entreprise investit efficacement dans son personnel durant chaque heure de son cycle d'exploitation, dès l'accueil (embauche jusqu'à la permanence), elle disposerait d'un capital humain compétitif pour réaliser sa croissance.

Pour tester les quatre hypothèses, nous avons emprunté la régression multiple avec entrée des variables de type pas-à-pas (stepwise). En effet, cette méthode de sélection des variables à inclure autorise la vérification de la variation du coefficient de détermination $\mathrm{R}^{2}$ de chaque variable explicative ajoutée à l'équation du modèle (Alain, 2004). Nous avons analysé les données à l'aide du logiciel SPSS 23.0. Le tableau 8 rend compte des résultats.

\subsubsection{Hypothèse 1. Les investissements en formation accroissent la compétence du capital humain}

Sur le plan qualitatif, les gestionnaires RH et responsables des cabinets sont unanimes sur l'importance de l'investissement en formation pour la construction $\mathrm{CHC}$ en milieu de travail :

[...] ici, la formation du personnel, dès son accueil et son intégration, est un droit reconnu par la convention collective. Nous offrons une formation en interne à tous les nouveaux employés, quel que soit le poste. Ensuite, dans notre budget, il existe une ligne dédiée à la formation; nous payons chaque année, le 1,8\% de la masse salariale au titre de la formation continue de notre personnel. Ainsi, nos salariés sont toujours aptes à exécuter leurs tâches de façon structurée et a permis ainsi de réaliser notre vision de leader. [...] nous avons élaboré un manuel dans lequel, la politique et le plan de formation continue du personnel sont définis, en collaboration avec le syndicat et avec l'appui de nos partenaires techniques (deux cabinets $\mathrm{RH}$, deux universités). Ensuite, nous avons signé une convention avec un bureau d'études en audit social qui assure deux missions par an. Et notre personnel est toujours doté de nouvelles connaissances en sécurité au travail, en nouvelle technologie de l'information, en assurance maladie et soins de premiers secours, etc.

Ces avis témoignent de l'efficacité de la construction du CHC par l'investissement en formation. Toutefois, quelques gestionnaires émettent des réserves quant à la finalité de la formation, toujours mal comprise par certains salariés :

[...] il y a deux ans, la réalisation de formation de notre personnel a généré des conflits énormes qui ont nécessité l'intervention du délégué de travail. Effet, tous ceux qui ont bénéficié de la formation réclament soit la promotion, soit une augmentation de salaire. C'est ce qui fait que depuis lors, la direction se méfie quant aux investissements en formation. 
Quantitativement, la formation prédit significativement le CHC (tableau 8). En effet, le coefficient de détermination $\mathrm{R}^{2}$, qui estime l'apport de chaque variable au modèle proposé, montre une contribution significative $\left(\mathrm{R}^{2}=0,21, p<, 00\right)$ de la formation au CHC. Autrement dit, la formation explique $21 \%$ de la variation de la construction du CHC dans les entreprises étudiées. Pour ce qui est du coefficient de régression $(b=0,19)$, il signifie qu'à chaque amélioration de l'échelle de la qualité de formation continue, le niveau du CHC de l'organisation prédit s'accroit. De même, le coefficient standardisé bêta $(\beta=0,23)$, qui exprime le coefficient de régression de la variable formation, montre que cette variable contribue à la prédiction du $\mathrm{CHC}$ et ce lorsque l'on considère un accroissement d'un écart-type des variables. La valeur du test $\mathrm{T}(t=8,62 ; p<, 00)$ indique que la contribution de la formation à l'explication du niveau du CHC de l'organisation est statistiquement significative. Ces résultats confirment l'hypothèse 1 portant sur la prédiction du CHC dans l'organisation par les investissements en formation.

\subsubsection{Hypothèse 2. Les pratiques d'encadrement du personnel améliorent la compétence du capital humain}

Qualitativement, il ressort de l'avis des responsables RH que :

[...] ici, les pratiques d'encadrement du personnel font partie intégrante de notre culture d'entreprise et débutent à la phase d'accueil pour favoriser l'intégration. Nous savons et sommes conscients que l'aptitude d'un nouveau salarié dépend du niveau de la qualité de l'encadrement que nous lui offrons. Par exemple, le service de la comptabilité a mis en place un dispositif à cet effet, il en est de même pour le service des ventes qui offre des encadrements collectifs et des ateliers à tous les agents de vente.

Chez nous, il y a toute une ligne budgétaire dédiée aux activités d'encadrement et d'accompagnement des nouveaux salariés qui commencent à l'étape d'accueil et se poursuivent jusqu'à l'intégration. Ce qui rend nos agents de vente, par exemple, très autonomes deux semaines, après leur prise de service. En conséquence, nous avons eu des résultats toujours positifs de nos agents. Ainsi, nos salariés sont toujours aptes à exécuter leurs tâches de façon structurée et a permis ainsi de réaliser notre vision de leader.

Ces avis témoignent de l'importance de l'encadrement comme instrument pertinent de la construction du CHC en milieu de travail à l'étape d'accueil.

Quantitativement, les pratiques d'encadrement prédisent aussi le CHC. En effet, selon les données du tableau 8, la valeur du $\mathrm{R}^{2}$ montre une contribution significative $\left(\mathrm{R}^{2}=0,48, p<, 00\right)$ de l'encadrement sur le CHC. Autrement dit, l'encadrement explique $27 \%$ supplémentaire de la variation de la construction du $\mathrm{CHC}$ dans les entreprises étudiées. Le coefficient de régression $(b=0,28)$ signifie qu'à chaque amélioration de l'échelle de la qualité de l'encadrement, le niveau du CHC s'accroît. Il en est de même pour le coefficient bêta $(\beta=0,23)$. La valeur du test $\mathrm{T}(t=8,72)$ indique que cette contribution à l'explication du niveau du $\mathrm{CHC}$ est significative. De tels résultats confirment l'hypothèse 2 .

\subsubsection{Hypothèse 3. Plus l'organisation crée des opportunités d'expérience, plus son capital humain serait compétitif}

Les résultats de l'entrevue montrent que les facteurs opportunités d'expérience et ancienneté accroissent l'aptitude du personnel à l'étape de la permanence.

[...] grâce à nos réseaux d'affaires, nous avons pu diversifier le capital expérience de notre personnel durant les deux dernières années tant sur l'aspect technique qu'administrative. Par exemple, novembre 2019, deux de nos collaborateurs de services santé et sécurité au travail ont effectué un voyage d'imprégnation de trois jours auprès de notre partenaire en charge de la sécurité santé an travail. À l'issue de cette visite, des 
ateliers d'information et de sensibilisation ont été réalisés au profit de tous les autres collaborateurs. Ainsi, aujourd'bui, le taux d'accidents de travail est considérablement réduit, je dirais de $80 \%$.

Certains responsables des RH, en insistant sur l'expérience comme stratégie pour rendre apte le personnel, ont mis de l'avant la contribution des partenaires techniques.

[...] la convention de soutien technique avec un bureau d'études en audit social a permis à notre entreprise de bien maîtriser et d'appliquer les principes de Responsabilité sociale des entreprises. Et, d'ici deux à trois ans, nous pourrions être certifiés.

Ces avis démontrent l'importance de la construction du CHC par les opportunités d'expérience durant leur étape de permanence.

Quantitativement, les opportunités d'expérience prédisent significativement le CHC. L'indicateur $\mathrm{R}^{2}$ présente une contribution significative $\left(\mathrm{R}^{2}=0,64, p<, 00\right)$ de l'expérience sur le CHC. Autrement dit, l'encadrement explique un supplément de $16 \%$ de la variation de la construction du CHC dans les entreprises étudiées. Le coefficient de régression $(b=0,31)$ signifie qu'à chaque amélioration de l'échelle de la qualité de l'encadrement, le niveau du CHC s'accroît. Il en est de même pour le coefficient bêta $(\beta=0,34)$ qui témoigne de cette même contribution. La valeur du test $\mathrm{T}(t=8,77)$ indique que cette contribution à l'explication du niveau du CHC est significative. De tels résultats confirment l'hypothèse 3.

\subsubsection{Hypothèse 4. L'environnement agile contribue à la construction du capital humain compétitif}

Qualitativement, les partisans de la GRH en milieu organisationnel sont d'avis que l'apprentissage collectif, l'auto-organisation et le travail d'équipe sont des facteurs de construction du capital humain compétitif.

[...] pour rendre nos collaborateurs agiles, nous faisons la promotion du travail en équipe, lequel fait partie de l'une de nos valeurs, car nous savons que ce mode de travail favorise l'apprentissage collectif, le partage d'expérience et la résolution des conflits. Grâce à la formation et l'opérationnalisation des équipes, nos collaborateurs accroissent notre performance en innovation organisationnelle et sociale depuis 2018.

Dans la même perspective, d'autres responsables reconnaissent que l'environnement agile favorise l'accélération des compétences en innovation du personnel durant l'étape de la permanence.

[...] moi, je ne suis pas spécialiste en innovation, mais selon les témoignages de certains de mes collaborateurs, il semble que le fait de leur accorderplus d'autonomie dans certains aspects de gestion favorise la créativité, mais aussi assure leur engagement en continu dans leurs postes et leurs missions. Et nos partenaires sociaux nous soutiennent dans cette approche...

Enfin, d'après le tableau 8 , l'agilité prédit aussi le CHC. Le coefficient de détermination $\left(\mathrm{R}^{2}=0,83, p<, 00\right)$ présente une contribution significative de l'agilité sur le CHC. Cela signifie que l'agilité explique $19 \%$ de variance additionnelle de la construction du $\mathrm{CHC}$ dans les entreprises étudiées. Le coefficient de régression $(b=0,29)$ signifie qu'à chaque amélioration de la qualité de l'agilité, le niveau du CHC augmente. De même, le coefficient bêta $(\beta=0,33)$ montre que l'agilité contribue à la prédiction du CHC. La valeur du test T $(t=8,92)$ indique que cette contribution à l'explication du niveau de CHC est significative. De tels résultats confirment l'hypothèse 4 .

En définitive, bien que chaque variable prédit significativement la construction du CHC, c'est la combinaison des quatre facteurs qui joue un rôle déterminant $\left(\mathrm{R}^{2}=0,83\right)$ dans la prédiction du CHC. Conséquemment, les quatre hypothèses sont toutes soutenues par les résultats. 
Tableau 8

Facteurs de prédiction du capital humain compétitif en milieu de travail

\begin{tabular}{l|c|c|c|c|c} 
Variables & $\mathbf{R}^{\mathbf{2}}$ & $\boldsymbol{b}$ & $\boldsymbol{\beta}$ & $\boldsymbol{t}$ & $\boldsymbol{p}$ \\
\hline Constante & & 2,17 & 2,37 & 8,62 &, 00 \\
\hline Formation & 0,21 & 0,19 & 0,23 & 8,72 &, 00 \\
\hline Encadrement & 0,48 & 0,28 & 0,32 & 8,77 &, 00 \\
\hline Expérience & 0,64 & 0,31 & 0,34 & 8,82 &, 00 \\
\hline Agilité & 0,83 & 0,29 & 0,33 & 8,92 &, 00
\end{tabular}

\subsection{Discussion}

L'étude visait à repérer les variables pour servir à construire et tester un modèle du capital humain compétitif en milieu organisationnel, durant l'étape d'accueil, d'intégration et de permanence, par des variables jugées pertinentes sur les plans théorique et pratique.

D’une manière générale, il ressort des résultats de cette étude que les pratiques d'entreprise étudiées, au moyen d'investissements en formation, de l'encadrement, de l'expérience et de l'environnement agile, engendrent le capital humain compétitif en milieu organisationnel dès l'étape d'accueil jusqu'à la permanence. Ces résultats sont significativement conformes aux travaux antérieurs (Becker, 2009; Nafukho et al., 2004; Poulain, 2001; Robinson et al., 2008; Schultz, 1961).

Spécifiquement, d'après les résultats qualitatifs et quantitatifs, la formation est associée, positivement et statistiquement significative, au capital humain compétitif (hypothèse 1) dès l'étape d'intégration. De même, elle améliore et contribue significativement à la prédiction du $\mathrm{CHC}$. De tels résultats ne sont pas fortuits. En effet, la formation, selon les propos de plusieurs théoriciens et praticiens, est un stimulant qui permet de répondre aux réactions et problématiques quotidiennes en milieu organisationnel (Lleras, 2004; OCDE, 1998; Yaroson et al., 2017). Ces résultats peuvent aussi s'expliquer par le fait que la formation est un moyen par lequel l'employé peut avoir la meilleure estime de soi, mais aussi reconnaître ses forces et ses talents pour l'organisation durant son séjour. Elle favorise, pour le salarié, l'augmentation du sentiment d'appartenance à son organisation; elle brise aussi les silos dans l'organisation. Pour l'organisation, la formation continue permet de retenir les meilleurs candidats. C'est un des éléments clés de la culture d'entreprise qui garantit sa posture compétitive (Mengistae, 2006). En conséquence, des investissements réguliers en formation continue constituent une stratégie pour parvenir à disposer du CHC au sein de l'organisation. Ces résultats sont similaires à ceux obtenus par plusieurs travaux antérieurs(Becker, 1962; Lleras, 2004; OCDE, 1998). En effet, selon les résultats de ces auteurs, les investissements en formation prédisent positivement et significativement le capital humain compétitif. Toutefois, certains de nos résultats qualitatifs empiriques convergent peu avec la littérature.

Ensuite, les pratiques d'encadrement influencent positivement le CHC (hypothèse 2) dès l'étape d'accueil et d'intégration. Un tel résultat n'est pas surprenant considérant que l'encadrement joue un rôle extrêmement important pour accompagner, communiquer auprès des salariés, mais aussi organiser la capitalisation. C'est ainsi que l'encadrement des nouvelles recrues contribue efficacement à la construction de leur capital (Santos, 2012; Yaroson et al., 2017).

De même, les opportunités d'expérience participent aussi à la construction du CHC dans l'organisation durant l'étape de la permanence. La relation qui émerge est positive et statistiquement significative comme suggéré par l'hypothèse 3. Ces expériences de travail sont souvent associées au capital humain compétitif selon plusieurs travaux portant sur la gestion des ressources humaines (Antonelli et al., 2010; Baranwal, 
2018; Robinson et al., 2008). Le fait que de tels résultats émergent pour les entreprises étudiées au Tchad prolonge ceux issus des autres contextes (Châteauneuf-Malclès, 2016; Chung, Park, Lee et Kim, 2015; Hayton, 2003).

Enfin, l'agilité organisationnelle favorise la construction du CHC, de l'intégration à la permanence. Un tel résultat n'est pas surprenant considérant que l'agilité joue un rôle extrêmement important pour rendre le personnel plus «intelligent», efficace et autonome. En outre, l'agilité, introduite comme variable de construction du $\mathrm{CHC}$, est une réponse stratégique et organisationnelle aux fluctuations environnementales. Nos résultats corroborent ceux de la littérature qui rapportent que l'agilité rime avec mobilité et réactivité (Legras, 2014; LeMay, 2019; Moinet, 2007). Les entreprises agiles sont en mesure de faire face, rapidement, mais efficacement, aux contraintes de l'environnement. De même, ces résultats s'expliquent aussi par le fait que l'agilité véhicule des valeurs et principes permettant aux équipes de travail de développer un langage partagé, mais aussi de faire émerger une intelligence collective et un mode de travail collaboratif (Legras, 2014; Moinet, 2007).

À la lumière de ce qui précède, nous pouvons souligner que l'impact de la formation, de l'encadrement, de l'expérience et de l'agilité sur le capital humain, lors de l'étape d'accueil à celle de la permanence, est indépendant de la situation économique du pays et des secteurs des entreprises étudiées. Il dépend plutôt des styles de management de l'entreprise qui sont favorables à l'enrichissement et au développement de la personne considérée comme un sujet de croissance et non comme une ressource de production. La deuxième explication associée à ces résultats est que le capital humain est un concept cognitif inséré dans un corpus théorique, mais aussi le résultat des pratiques sur le terrain de manœuvre quotidienne des activités de l'entreprise. Comme le montre si bien nos résultats, le modèle suggéré est un modèle de l'action humaine construit de telle manière qu'une initiative (formation, encadrement, etc.), au profit de la personne au sein de l'organisation, soit compréhensible aussi bien pour l'acteur lui-même que pour le bénéficiaire et ses semblables (Becker, 1962, 2009; Lleras, 2004). Cette action s'inscrit dans le cadre d'interprétation courante de la vie quotidienne de la personne comme sujet de croissance de l'entreprise (Schultz, 1961).

Sur la base des discussions qui émergent avec des points de vue convergents, divergents et complémentaires, on peut envisager des implications théoriques, méthodologiques et managériales, souligner les limites et suggérer des avenues. C'est l'objet des lignes qui suivent.

\section{Contribution de la présente étude}

\subsection{Contribution théorique}

Notre article se propose de contribuer à l'avancement des études sur la valorisation du capital humain en milieu de travail dès l'étape d'accueil. Les facteurs mis de l'avant par les travaux scientifiques antérieurs sont, majoritairement, liés aux pratiques de management. Tandis que les relations testées entre les pratiques de management, notamment la formation et le capital humain compétitif, sont des relations directes. Les résultats desdits travaux antérieurs ont débouché sur la conclusion qu'une formation qualifiante continue et l'expérience participent efficacement à la construction du capital humain compétitif en milieu de travail. Mais les variables telles que l'encadrement et l'agilité n'ont pas été, explicitement, intégrées dans les modèles. Aussi, la définition formelle explicite du capital humain $(\mathrm{CH})$ n'a pas été proposée. Ainsi, la principale contribution théorique de cette étude est d'avoir introduit, dans le modèle théorique, l'encadrement et l'agilité comme variables de construction du capital humain compétitif en milieu de travail.

C'est surtout la combinaison de ces quatre variables qui constitue la contribution théorique majeure. De même, nous avons proposé une définition du $\mathrm{CH}$ dont le besoin d'amélioration demeure toujours. 
Nous avons montré que dans le processus de construction du capital humain en milieu de travail, l'environnement agile et l'encadrement ont un fort pouvoir explicatif du meilleur capital humain. Autrement dit, la promotion du management collaboratif, l'autonomisation des travailleurs ainsi que les initiatives d'accompagnement et de soutien au personnel jouent un rôle déterminant dans le cheminement de la production du personnel habile. Inversement, un style de management mécanique inhibe l'épanouissement du capital humain. Cette recherche vient montrer qu'il n'y a pas que la formation qui peut produire le capital humain compétitif.

Outre les apports ci-dessus, cette étude fait émerger de nouvelles avenues théoriques. En effet, l'identification et le test de nouvelles variables, notamment «environnement agile» et «l'accompagnement» constituent la contribution à la valorisation de l'innovation du capital humain en milieu de travail. Cette démarche, dont le modèle suggéré en est la manifestation, n'avait pas fait l'objet d'une étude ayant déployé un devis qualitatif et quantitatif rigoureusement empirique. Il ressort ainsi que le capital humain en milieu organisationnel, pour qu'il soit compétitif, nécessite de l'investissement spécifique dans les pratiques de management collaboratif/coopératif. En conséquence, les deux principales avenues théoriques suivantes sont dans le collimateur: (i) théorie de management coopératif et; (ii) théorie de management participatif. Les partisans (Jacquet, 2013; March et Olsen, 1984; Ouchi et Chung, 1982) de ces courants de pensée mettent de l'avant l'accent sur la formation, le bien-être au travail et l'importance de l'organisation comme système ouvert avec une vision sociale pour parvenir à être plus compétitive.

\subsection{Contribution méthodologique}

D’une manière générale, l'adoption de la méthode mixte simultanée (qualitative et quantitative) constitue la contribution méthodologique majeure. Spécifiquement, l'adoption des nouveaux construits et l'adaptation des contenus des items formant les dimensions des variables utilisées dans le contexte de cet article représentent un apport méthodologique remarquable, car les études précédentes n'ont pas pris en compte les constituantes spécifiques à l'objet de la recherche. En effet, l'étude hypothético-déductive menée a permis de mettre en évidence des items propres à notre contexte d'étude.

Ensuite, après analyse corrélationnelle et explicative, plusieurs items se sont révélés fiables et pertinents. Il s'agit, entre autres, de : (i) la flexibilité organisationnelle; (ii) le soutien des nouvelles recrues; (iii) la tolérance aux risques; (iv) la politique de formation et; (v) l'autonomie organisationnelle. Il s'agit des items qui ont été formulés par nos soins dans le cadre de ce travail. Ainsi, la fiabilité et la pertinence de ces items s'inscrivent dans l'éventail des apports méthodologiques remarquables.

Enfin, le devis mixte déployé constitue un apport méthodologique indéniable. En effet, cette stratégie a permis de minimiser les limites que montrent les démarches qualitative et quantitative. C'est une stratégie qui a permis d'avoir une compréhension plus fine et complète de la problématique de construction du capital humain compétitif au travail. Cette démarche s'est déployée en une phase au cours de laquelle les données qualitatives et quantitatives ont été générées simultanément et de manière itérative. Par ailleurs, l'analyse côte à côte de la combinaison des démarches qualitative et quantitative a permis de documenter les concepts/variables peu étudiés, notamment « agilité » et « expérience ».

\subsection{Contributions managériales}

L'identification des variables encadrement et agilité, qui jouent un rôle significatif dans la construction du capital humain compétitif en milieu de travail, représente une contribution concrète pour tous les acteurs impliqués dans le processus de valorisation du capital humain. En effet, la mise en lumière des relations positives et statistiquement significatives, entre la formation continue, l'expérience, l'accompagnement, l'agilité et le capital humain compétitif, est un élément important sur lequel les gestionnaires du personnel, 
les consultants et les chercheurs en GRH peuvent s'appuyer pour suggérer et enrichir les pratiques de management du capital humain.

De même, la présente étude a permis de mettre en évidence le pouvoir explicatif de l'environnement agile, l'accompagnement et la formation sur le capital humain compétitif. Pour la formation, en revanche, les résultats ont montré que certaines organisations y sont peu favorables. Cependant, étant donné la caractéristique brutale et accélérée de l'écosystème socioéconomique actuel, cette étude recommande aux gestionnaires des RH de mettre davantage l'accent sur la formation en lien avec l'usage des réseaux sociaux, de l'outil informatique, mais également l'accompagnement et les méthodes agiles dans leur pratique de management du capital humain. De manière spécifique, il est souhaitable que l'accent soit mis sur les différentes dimensions de l'agilité et donc de l'intelligence collective pour favoriser l'épanouissement du capital humain.

Quoi qu'il en soit, ce travail a permis de montrer que la combinaison entre formation, expérience, encadrement et agilité joue un rôle plus significatif sur la construction du capital humain compétitif que l'emploi différencié. Ce résultat est fondamental pour les chefs d'entreprise qui devraient faire face à la concurrence, à l'environnement brutal et en perpétuel changement.

\section{Limites et orientations futures}

Outre les contributions, cette étude présente également des limites. Premièrement, les résultats de notre étude s'appuient sur des informations fournies par un échantillon de 178 parties prenantes à la GRH, dans le contexte de la nouvelle économie. Par rapport à plusieurs autres études menées sur le capital humain, notre recherche a conduit au même résultat, à savoir que la relation entre les pratiques de management (formation, encadrement, expérience et agilité) et le $\mathrm{CHC}$ est positive et statistiquement significative. Mais ce résultat doit, toutefois, être considéré avec réserve lorsqu'il est généralisé à d'autres contextes. À notre avis, le résultat dépend beaucoup plus du contexte interne de l'entreprise et de l'échantillon sélectionné. Il semble donc utile d'étudier à la fois plus de trois échantillons d'entreprises : des échantillons caractérisés par des politiques sociales développées et des échantillons où les pratiques d'entreprise sont peu visibles. La deuxième limite est que l'axe de construction du $\mathrm{CHC}$ a été étudié dans son aspect statique, dans la mesure où les répondants, majoritairement des chefs de service, sont invités à fournir leur appréciation sur les initiatives développées par leur entreprise. Ainsi, une approche longitudinale, intégrant le personnel issu de plusieurs niveaux hiérarchiques, s'avère nécessaire dans les recherches futures. En outre, plusieurs sujets intéressants sont laissés en plan pour de futures recherches. Cette étude nous laisse convaincus que les investissements en formation, les pratiques d'encadrement, l'environnement agile, etc. accroissent la construction d'un $\mathrm{CHC}$ dans certaines conditions dès la phase d'accueil; ce qui a été démontré par notre recherche. Les recherches futures devraient accorder plus d'attention à cette question tout en introduisant d'autres variables, telles que l'innovation dans ses différentes dimensions et les variables modératrices/médiatrices, car notre étude a testé la relation directe entre le $\mathrm{CHC}$ et la formation, l'accompagnement, l'agilité, etc.

\section{Conclusion}

Il y a lieu de répondre à notre question de recherche initialement formulée à savoir «quelles sont les variables jugées pertinentes pour élaborer le meilleur modèle du $\mathrm{CH}$ au sein d'une organisation? D'entrée de jeu, l'exploration de la littérature a permis de tracer explicitement la trajectoire de quatre variables pertinentes ayant permis de suggérer le modèle de construction du $\mathrm{CH}$ en milieu de travail. Ensuite, nos résultats empiriques ont montré, qu'outre la formation et l'expérience, l'accompagnement/encadrement et l'environnement agile ont montré significativement leur pouvoir explicatif sur le processus de construction du capital humain compétitif (étapes d'accueil, d'intégration et de permanence) en milieu de travail. C’est 
l'introduction des variables encadrement/soutien et environnement agile qui ont mis en lumière la construction du capital humain. Cependant, c'est surtout la combinaison de ces quatre facteurs qui joue un rôle déterminant dans la fertilisation de l'habileté du personnel en milieu de travail. Toutefois, il convient de noter que l'étude est menée sans tenir compte des facteurs contingents, notamment de l'écosystème et des variables modératrices/médiatrices.

Quoi qu'il en soit, les résultats de notre étude conduisent à penser que placer la problématique de la valorisation du capital humain au centre de la mission de l'entreprise aujourd'hui est une question de responsabilité et non de liberté. Ainsi, le modèle proposé est un objet de pensée qui contribuerait à enrichir les actions des $\mathrm{RH}$, mais aussi à déterminer le comportement du gestionnaire du $\mathrm{CH}$, à définir le but de ses actions et à prescrire les moyens pour les réaliser (Schultz, 1961). Par ailleurs, il s'agit, pour ainsi reprendre les propos de Schultz (1987), d'une construction au deuxième degré, car le capital humain est, à la fois, un concept cognitif, inséré dans un corpus théorique et une catégorie de la pratique.

En outre, la construction du capital humain en milieu du travail est une question de responsabilité, mais aussi de devoir, dans le sens qu'aucune organisation ne devrait craindre de s'y engager, quelles que soient sa taille et ses ressources. Si elle investit consciencieusement dans la formation, les opportunités d'expérience, les initiatives d'encadrement et l'amélioration du style de management, elle peut réaliser de meilleures performances et figurer parmi les entreprises ayant le capital humain le plus compétitif dans un environnement concurrentiel.

En définitive, il convient de relever l'importance d'aller beaucoup plus loin dans les investigations sur la construction du capital humain compétitif en milieu organisationnel et sur les pratiques de management les plus pertinentes pour sa valorisation. La présente recherche a besoin d'une meilleure compréhension des pratiques de valorisation du capital humain dans le monde actuel caractérisé par l'économie d'innovation et non de rattrapage. Quoi qu'il en soit, les attentes majeures sont relatives aux politiques de formation, aux modes d'encadrement/accompagnement ainsi qu'à la création et à l'enrichissement d'un environnement agile pour favoriser la valorisation du capital humain. Cette étude fait émerger un domaine toujours à fort potentiel de recherche dont les attentes en matière de conceptualisation sont toujours peu satisfaites.

\section{RÉFÉRENCES}

Ahmad, S. b. et Mushraf, A. M. (2011). The Relationship between Intellectual capital and Business Performance: An empirical study in Iraqi industry. International Conference on Management and Artificial Intelligence (2011), 1-6.

AL-Duajaili, M. A. A. (2012). Influence of Intellectual Capital in the Organizational Innovation. International Journal of Innovation, Management and Technology. 3(2), 128-135.

Anie-Tchad. (2017). Guide d'Installation des Entreprises au Tchad. wnw.anie-tchad.com, 2017, 47.

Antonelli, G., Antonietti, R. et Guidetti, G. (2010). Organizational change, skill formation, human capital mesurement: evidence from italian manufacturing firm. Journal of Economic Surveys, 24(2), 206-247.

Baranwal, G. (2018). Links between foreign direct investment and human capital formation: Evidence from the manufacturing sector in India. The Journal of International Trade \& Economic Development, 1-24. doi:10.1080/09638199.2018.1508304

Barney, J. B. (1991). Firm Resources and Sustained Competitive Advantage. Journal of Management, 17, 99-120.

Becker, G. S. (1962). Investment in Human Capital: A Theoretical Analysis. Journal of Political Economy. 70(5), 9-49.

Becker, G. S. (2009). Human capital: A theoretical and empirical analysis, with special reference to education University of Chicago Press. 
Bessieux-Ollier, C. et Walliser, É. (2010). Le capital immatériel État des lieux et perspectives. Revue française de gestion 2010(207), 85 à 92.

Bosma, N., Praag, M. v., Thurik, R. et Wit, G. d. (2002). The Value of Human and Social Capital Investments for the Business Performance of Startups. EIM Business and Policy Research, 1-20.

Bouteiller, D., Cossette, M., Fournier, C. et Sabourin, N. (2013). Capital humain et performance(s). Cadres théoriques et protocoles de mesure. HEC Montréal, 2013, 241.

Chamak, A. et Fromage, C. (2006). Le capital humain. Editions Liaisons.

Châteauneuf-Malclès, A. (2016). LE CAPITAL HUMAIN, une source de compétitivité délaissée ? Idées économiques et sociales 2(184), 25-34.

Chung, C. C., Park, H. Y., Lee, J. Y. et Kim, K. (2015). Human capital in multinational enterprises: Does strategic alignment matter? Journal of International Business Studies, 2015(46), 806-829.

Creswell, J. (2013). Research Design: Qualitative, Quantitative, and Mixed Method Approaches, 4th. Sage, 4(2013), 1-285.

Crook, T. R., Combs, J., Todd, S., Woehr, D. et Ketchen, J. (2011). Does human capital matter? A meta-analysis of the relationship between human capital and firm performance. Journal Application Psychologic, 96(3), 443-456.

Evrard, Y., Pras, B., Roux, E., Desmet, P., Dussaix, A.-M. et Lilien, G. (2009). Market - Fondements et méthodes de recherches en marketing.

Fraisse-D'Olimpio, S. (2009). Les fondements théoriques du concept de capital humain (Partie 1). OCDE, 2009.

Hayton, J. C. (2003). Strategic human capital management in SMEs: an empirical study of entrepreneurial performance. Human Resource Management., 42(4), 375-391.

Jacquet, S. (2013). Du management participatif...au management coopératif : coopérer pour construire et donner du sens au management. creg.ac.versailles, 2013, 1-17.

Kalkan, A., Bozkurtb, Ö. Ç. et Arman, M. (2014). The impacts of intellectual capital, innovation and organizational strategy on firm performance. Procedia - Social and Behavioral Sciences, 150(2014), 700 - 707.

Keeley, B. (2004). Le capital humain: comment le savoir déterminer ? Les essentiels de l'OCDE, 163.

Kungwansupaphan, C. et Siengthai, S. (2012). Exploring entrepreneurs' human capital components and effects on leaming orientation in early internationalizing firms. International Entrepreneurship and Management Journal of, 2012, 1-27.

Legras, S. (2014). L'agilité, nouvelle transformation pour l'entreprise. Documentaliste-Sciences de l'Information, 51(4), 4-6.

LeMay, M. (2019). Agile for Everybody. O’Reilly Media, 2019, 135.

Lev, B. et Han, D. (2015). The Relationship between Intellectual Capital and Corporate Performance in Chinese Bio-pharmaceutical Industry. SHS Web of Conferences., 0102(2015), 1-7.

Lleras, M. P. (2004). Investing in Human Capital: A Capital Markets Approach to Student Funding. Cambridge University Press, New York, $248 \mathrm{p}$.

March, J. et Olsen, J. (1984). The New Institutionalism: Organizational Factors in Political Life. The American Political Science Review, 78(3), 734-749.

Mengistae, T. (2006). Competition and entrepreneurs' human capital in small business longevity and growth Taye Mengistae. The Journal of Development Studies, 42(5), 812-836.

Moinet, N. (2007). L'agilité stratégique : une question de dispositif intelligent, Vie és sciences de l'entreprise, 2007(174-175), 142-155.

Nafukho, F. M., Hairston, N. et Brooks, K. (2004). Human capital theory: implications for human resource development. Human Resource Development International, 7(4), 545-551. 
OCDE. (1998). L'investissement dans le capital humain : Une comparaison internationale. OCDE, 1998, 121.

OCDE. (2013). Nouvelle source de croissance : le capital intellectuel, analyses de base et conclusion pour l'action gouvernementale. OCDE, 2013, 73 .

Ouchi, W. et Chung, K. (1982). Theory Z: How American Business Can Meet the Japanese Challenge. The Academy of Management Review, 7(2), 317-319.

Oxley, L., Lee, T. et Gibson, J. (2008). Measuring human capital: Alternative methods and international evidence. THE KOREAN ECONOMIC REVIEW, 24(2), 63.

Poulain, É. (2001). Le capital humain, d'une conception subtantielle à un modèle représentationnel. Revue économique, 52(1), 91-116.

Robinson, D., Hooker, H. et Mercer, M. (2008). Human Capital Measurement: Approaches, issues and case studies. Workforce Planning Guide, 2008, 86.

Rodriguez, J. et Loomis, S. (2007). A new view of institutions, human capital, and market standardisation. Education, Knowledge and Economy, 1(1), 93-105.

Rodriguez, J. P. et Loomis, S. R. (2007). A new view of institutions, human capital, and market standardisation. Education, Knowledge and Economy, 1(1), 93-105. doi:10.1080/17496890601128357

Santos, M. E. (2012). Human Capital and the Quality of Education in a Poverty Trap Model. Oxford Development Studies, 39(1), $25-47$.

Schultz, T. (1961). Investment in Human Capital. The American Economic Review, 51(1), 1-17.

Stiglitz, J. E., Greenwald, B. C., Aghion, P., Arrow, K. J., Solow, R. M. et Woodford, M. (2014). Creating a Learning Society: A New Approach to Growth, Development, and Social Progress. Columbia University Press, 2014.

Ugalde-BindaI, N., Balbastre-Benavent, F., Canet-Giner, T. et Escribá-Carda, N. (2013). The role of intellectual capital and entrepreneurial characteristics as innovation drivers. Innovar, 24(53), 41-60.

Unger, J., Rauch, A., Frese, M. et Rosenbusch, N. (2011). Human capital and entrepreneurial success: A meta-analytical review. Journal of Business Venturing, 26(2011), 341-358.

Vinokur, A. (2014). Pourquoi le "capital" humain ? L'Harmattan. (2014), 1-11.

Yaroson, E. V., Esew, N. G. et Abdul-Qadir, A. B. (2017). Human capital development in Nigeria: an empirical assessment on the impact of corruption. African J. Economic and Sustainable Development, 6(1), 86-103. 